\title{
CONTROL OF DIFFUSE VACUUM ARC USING AXIAL MAGNETIC FIELDS IN COMMERCIAL HIGH VOLTAGE SWITCHGEAR
}

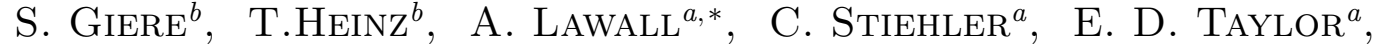 \\ S. WETHEKAM ${ }^{b}$ \\ ${ }^{a}$ Siemens AG, Smart Infrastructure, Rohrdamm 88, 13623 Berlin, Germany \\ ${ }^{b}$ Siemens AG, Gas and Power, Nonnendammallee 104, 13623 Berlin, Germany \\ * andreas.lawall@siemens.com
}

\begin{abstract}
During the development of a commercial vacuum interrupter for application in HV switchgear at a rated voltage of $145 \mathrm{kV}$, we investigated the behavior of vacuum arcs controlled by axial magnetic fields (AMF). AMF arc control is already extensively used in medium voltage $(1-52 \mathrm{kV})$ applications, the key difference is the 2-3 times larger contact gap and the corresponding reduction of the AMF strength for HV applications. We conducted several stress tests with short circuit currents up to $40 \mathrm{kA}$, thus not only testing the interrupting capability, but also the electrical endurance of such a contact system. We also investigated the dielectric behavior of the vacuum interrupter by testing the capacitive switching duty. Despite the large number of operations, the contacts showed a minimal amount of contact erosion and damage and demonstrated behavior very similar to the extensive experience with MV vacuum interrupters. In line with simulation results, we conclude that even at high contact gaps and currents, a diffuse vacuum arc was maintained which distributed the arc energy evenly over the contacts.
\end{abstract}

Keywords: High Voltage, Vacuum Interrupter, Vacuum Arcs.

\section{Introduction}

Vacuum interrupters (VI) have been used in high voltage (HV) applications above $52 \mathrm{kV}$ from the start of practical VI manufacturing in the late 1950's and early 1960's. Early circuit breaker designs successfully achieved ratings up to $145 \mathrm{kV} / 40 \mathrm{kA}$ [1] and $132 \mathrm{kV} / 3500$ MVA [2] in type tests, and achieved some limited field use. However, the circuit breakers were extremely complex, using multiple VI in series [1], often with complex voltage grading systems [2]. The complexity and cost of these circuit breakers limited their use to very isolated examples. A concerted focus on HV VI development in Japan resulted in the field installation of approximately 8300 circuit breakers using VI from the late 1970's to 2010 [3]. 99\% of these circuit breakers were for $72 \mathrm{kV}$ and lower system voltages. Many of these designs, especially in later years, used a single VI or at most two VI in series instead of the previous multiple VI systems. Nevertheless, even in Japan only a very small percentage of the installed base of HV circuit breakers used vacuum.

In the 2000's, a broader interest in the use of VI for HV circuit breakers arose, primarily driven by legal burdens and restrictions on the use of $\mathrm{SF}_{6}$. This renewed focus centered on applications for system voltages of $110-170 \mathrm{kV}$. Interest appeared in Europe $[4,5]$, China [6], and Korea [7]. These were primarily R\&D projects; however, a series of type tests were performed on a single-break VI design for $126 \mathrm{kV} / 40 \mathrm{kA}$ from a Chinese university, which however did use $\mathrm{SF}_{6}$ as an external insulation [8].
Very early in the development of VI, it was thought that holding off the voltage would be straightforward, but current interruption would be difficult. This resulted in some early designs for $>72 \mathrm{kV}$ applications as load break switches and capacitor switches [9]. Instead, while high current interruption was manageable, enabling generator breaker applications up to $100 \mathrm{kA}$ [10] and several specialized designs for high currents up to $200 \mathrm{kA}$ [11-13] application to voltages above the medium voltage (MV) range faced several challenges. One fundamental problem was the general dependence of the breakdown voltage on the contact gap, which scales as roughly the square root of the contact separation [14]. In the MV range, this leads to very moderate contact gaps that are very compatible with various arc control techniques using the magnetic field generated by the short-circuit current $[14,15]$. HV (high voltage) applications, particularly for system voltages $>=110 \mathrm{kV}$, require longer contact gaps that creates challenges for arc control [16] and prompted consideration of sophisticated contact opening parameters [17]. In addition, a high voltage across an open metal contact gap in vacuum can produce $\mathrm{x}$-ray emission, with increasing risk of $\mathrm{x}$-ray emission as the voltage increases [18].

A concerted effort lead to the successful complete type testing of a commercial $\mathrm{SF}_{6}$-free vacuum circuit breaker for $145 \mathrm{kV} / 40 \mathrm{kA}$. The tests included verification of the complete range of requirements for practical circuit breaker applications. This achievement built on considerable efforts in the field experience of $72 \mathrm{kV}$ circuit breakers with VI [19], control of x-ray emission 


\begin{tabular}{lccc}
\hline \hline & Arc voltage & Normal current & Short-circuit breaking current \\
\hline Vacuum, AMF-contact & $30-40$ Volts & 10,000 operations & 50 operations \\
$\mathrm{SF}_{6}$, self-compression & Several Kilovolts & 6,000 operations & 12 operations \\
\hline \hline
\end{tabular}

Table 1. Comparison of typical breaking performance for vacuum and $\mathrm{SF}_{6}$.

from HV VI [20], and modeling of the arc behavior [16].

\section{Experiments}

The 3AV1 passed all relevant tests to ensure the reliability of a fully type tested product.

In comparison to a gas circuit breaker, the stress on vacuum interrupter contacts during switching operations with normal and short-circuit currents is significantly lower. The short arcing times and the low arc voltage of vacuum interrupters help to reduce the contact wear inside of the vacuum interrupter.

This results in a significantly higher number of breaking operations for vacuum interrupters compared to gas circuit breakers, enabling the design of vacuum interrupters capable to switch currents of several kA up to a million times, e.g., in tap changer applications [14].

In order to determine the high switching capability of our commercial vacuum interrupter for $145 \mathrm{kV} / 40 \mathrm{kA}$, we have performed arc voltage and arcing times investigations in extended high-power test series. In the latter, we demonstrate that the stresses of several high-power type tests can be handled with a single vacuum interrupter, and the electrical endurance performance is superior to established commercial high-voltage $\mathrm{SF}_{6}$ switchgear.

Table 1 compares the arc voltage during a short circuit current interruption using our $145 \mathrm{kV}$ highvoltage vacuum and a commercial $145 \mathrm{kV} \mathrm{SF}_{6}$ gas circuit breaker. Whereas the intensively cooled plasma column of a $\mathrm{SF}_{6}$ circuit breaker produces several kilovolts of arc voltage, the vacuum arc voltage is of the order of some $10 \mathrm{~V}$ only, resulting in a two orders of magnitude lower arc energy dissipated in the interrupter unit of the circuit breaker.

In Fig. 1, we compare minimum arcing times of our tests with a commercial $145 \mathrm{kV} \mathrm{SF}_{6}$ gas circuit breaker with our $145 \mathrm{kV}$ vacuum circuit breaker for a number of high-power test duties according to international standards (e.g. IEC 62271-100, etc.). Whereas for the $\mathrm{SF}_{6}$ circuit breaker, minimum arcing times of $12-$ $16 \mathrm{~ms}$ are observed, the minimum arcing times for the vacuum circuit breaker are in the range of 4-6 ms only. This results in significantly shorter arc durations for our vacuum circuit breaker, i.e. a second effect reducing the arc energy impact on the contact system of the interrupter unit compared to $\mathrm{SF}_{6}$ gas circuit breakers.

In our experiments, we found that these effects result in pratically neglibible erosion (see section 3 ) of

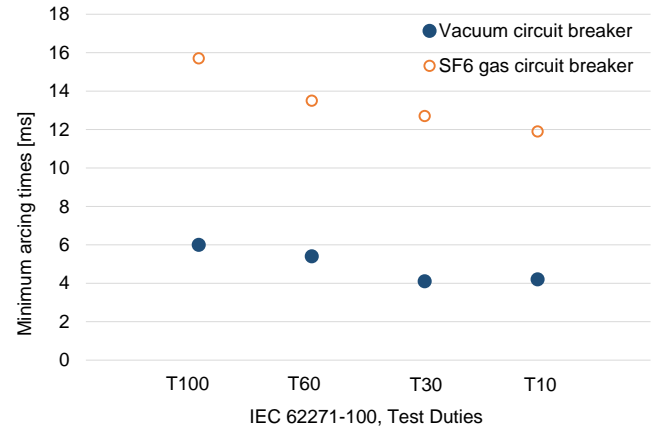

Figure 1. Comparison of minimum arcing times between $\mathrm{SF}_{6}$ and vacuum in type test duties.

\begin{tabular}{ccr}
\hline \hline test duty & Current $[\mathrm{kA}]$ & Operations \\
\hline T10 & 4 & 9 \\
T30 & 12 & 7 \\
T60 & 24 & 9 \\
L75 & 30 & 8 \\
OP2 & $6-10$ & 9 \\
T100a & 40 & 4 \\
T100s(b) & 40 & 13 \\
Verification & 40 & 3 \\
Integrity Check & $6-17$ & 3 \\
\hline Sum & & $\mathbf{6 5}$ \\
\hline \hline
\end{tabular}

Table 2. Summary of test duties conducted with a single vacuum interrupter.

the vacuum interupter unit during type test for highvoltage circuit breakers according to international standards. As an example, during the development of our $145 \mathrm{kV} / 40 \mathrm{kA}$ vacuum circuit breaker, we have performed the series of type tests with a single vacuum interruper given in Table 2. We performed 65 highpower tests including 17 tests T100a and T100s, which represent the highest thermal stress for the contacts of the standard type tests according to interantional standards.

For commercial $145 \mathrm{kV}$ self-compression gas circuit breakers, the contact system and nozzle show a pronounced erosion during switching operations with rated short-circuit currents, so that the interrupter unit needs to be replaced after about 12 operations. As indicated in Table 1, the number of high-current operations for our vacuum interrupter in the high-power test series was significantly higher. 


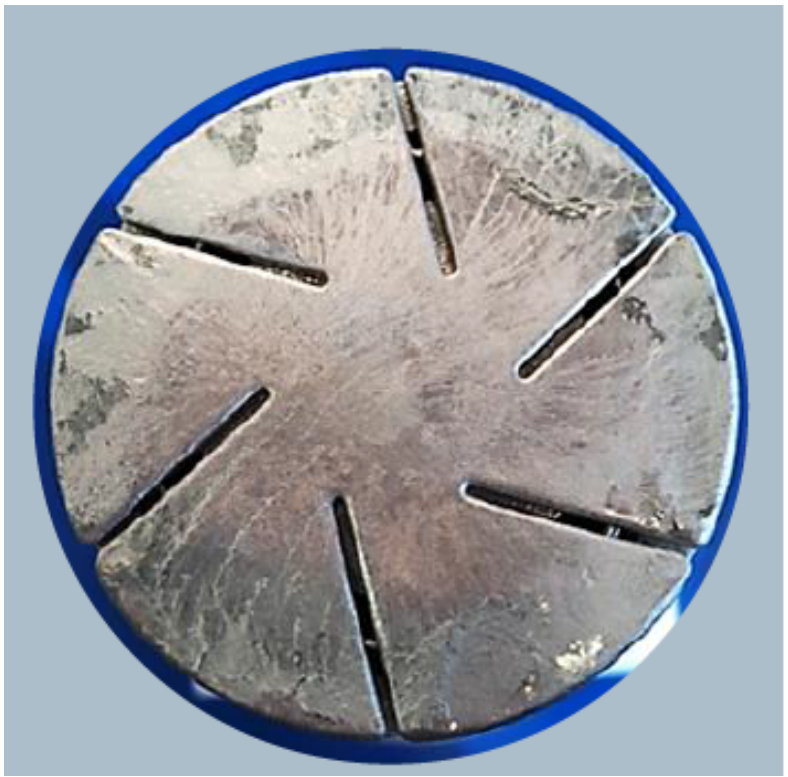

Figure 2. Picture of one contact surface of the opened $V I$ after all current interruptions.

We have also successfully performed line- and cablecharging capacitive switching tests with a capacitive voltage factor $k_{\mathrm{c}}=1.4$ where we achieved the highest capacitive switching class (very low probability of restrike) C2. This demeonstrated that high voltage vacuum circuit breakers are not limited to capacitive switching class C1 [8].

All tests were concluded with verfication tests, integrity tests and condition checks according to the specific international standard being tested (eg. IEC 62271-100, etc.).

\section{Experimental Results}

After all tests, which were part of the realized type test procedure, the vacuum interrupter was opened and inspected. Figure 2 shows a picture of the contact plate surface. We found that the contacts suffered almost no wear which indicates that their lifetime is significantly higher than tested in our extended test series.

A suitably dimensioned AMF contact system is essential for low contact erosion and ensures a high number of successful breaking operations. In summary, we can state that for appropriately dimensioned AMF contacts contact wear seem to play an almost negligible role. After the thermal load for the confirmed breaking operations, the loss of contact material is negligible. Therefore, we can conclude that contact wear of $145 \mathrm{kV}$ vacuum interrupters does not need to be monitored during the electrical lifetime of the circuit breaker.

\section{Simulation Results}

The simulations are based on a two-temperature magneto-hydrodynamic model of the arc plasma. The AMF produced by the current path in the contacts

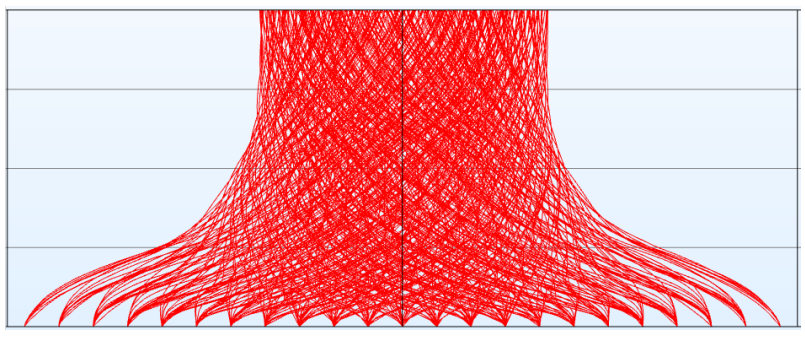

Figure 3. Plot of the streamlines of the ion velocity of ions emerging from the cathode (side-view). The cathode is located at the bottom, the anode is located at the top. Shown is the full contact diameter and contact gap.

is first calculated using standard FEM-tools. Then a smoothed approximation of this AMF is used as input for the plasma model. Details of this model can be found in Refs [21] and [22].

The arc plasma is simulated within a cylindrical volume between a pair of disc-shaped electrodes. Plasma length and disc diameter correspond to the contact gap length and the contact diameter of our commercial circuit breaker. The applied current was $40 \mathrm{kA}$ (RMS).

Figure 3 shows a side-view of the streamlines of the ions emerging from the cathode. Although a slight contraction of the arc can be seen in this result it still remains in a diffuse state. For the design of the contacts it is crucial to establish enough AMF to keep at least such a partly diffuse state of the arc. So the simulation model proved to be a useful tool to explore the contact designs for the high gaps needed in this application.

\section{Conclusion}

Vacuum interrupters are the dominant technology for circuit breakers in medium voltage applications based on the long electrical life at load and shortcircuit currents, as well as being maintenance-free and offering minimal contact erosion during operation. Issues with the relatively long contact gap required for $\mathrm{HV}$ applications initially prevented the penetration of vacuum into $\mathrm{HV}$ circuit breakers and switches, aside from a few specialized applications. Extensive efforts in arc modeling guided the successful development of AMF arc control techniques for these long contact gaps. Type testing verified the performance of VI for practical HV circuit breaker applications. Further high-power testing demonstrated that VI in HV keep the advantages seen in MV applications, such as very long electrical life and minimal contact erosion.

\section{Acknowledgements}

We like to thank Dr. Norbert Wenzel and Dr. Andreas Geisler for their support concerning the numerical arc model. 


\section{References}

[1] P. G. Slade et al. The development of a vacuum interrupter retrofit for the upgrading and life extension of $121 \mathrm{kV}-145 \mathrm{kV}$ oil circuit breakers. IEEE Trans. Power Del., 6(3):1124/1131, 1991. doi:10.1109/61.85857.

[2] L. Falkingham and M. Waldron. Vacuum for HV applications - perhaps not so new? - thirty years service experience of $132 \mathrm{kV}$ vacuum circuit breaker. Proc. 22nd ISDEIV, 1:200-203, 2006. doi:10.1109/DEIV.2006.357267.

[3] R. Smeets et al. The impact of the application of vacuum switchgear at transmission voltages. Cigre Working Group A3.27; Cigre Technical Report, (589), 2014.

[4] R. Renz. High voltage vacuum interrupters - technical and physical feasibility versus economical efficiency. Proc. 22nd ISDEIV, 1:257-262, 2006. doi:10.1109/DEIV.2006.357281.

[5] X. Godechot, S. Chakraborty, A. Girodet, and P. Vinson. Design and tests of vacuum interrupters for high voltage circuit breakers. Proc. 26th ISDEIV, 1:417-420, 2014. doi:10.1109/DEIV. 2014.6961708.

[6] Z. Liu, J. Wang, S. Xiu, Z. Wang, S. Yuan, L. Jin, H. Zhou, and R. Yang. Development of high-voltage vacuum circuit breakers in China. IEEE Trans. Plasma Sci, 35(4):856-865, 2007.

doi:10.1109/TPS.2007.896929.

[7] J. Ryu, Y.-G. Kim, J. Choi, and S. Park. The experimental research of $170 \mathrm{kV}$ VCB using single-break vacuum interrupter. Proc. 25th ISDEIV, 2:493-496, 2012. doi:10.1109/DEIV. 2012.6412563.

[8] X. Yao, J. Wang, Y. Geng, J. Yan, Z. Liu, J. Yao, and P. Liu. Development and type test of a single-break $126-\mathrm{kV} / 40-\mathrm{kA}-2500-\mathrm{A}$ vacuum circuit breaker. IEEE Trans. Power Del., 31(1):182, 2016. doi:10.1109/TPWRD . 2015.2456033.

[9] H. C. Ross. Vacuum power switches: 5 years of field application and testing. Trans. AIEE Part III: Power App. Syst., 71:758, 1961.

doi:10.1109/AIEEPAS.1961.4501132.

[10] H. Urbanek, K. R. Venna, and N. Anger. Vacuum circuit breakers - promising switching technology for pumped storage power plants up to 450 MVA. Proc. ICEPE 4th Int. Conf. Electr. Power Equip. - Switching Technol., page 107, 2017.

[11] S. Yanabu, S. Souma, T. Tamagawa, S. Yamashita, and T. Tsutsumi. Vacuum arc under an axial magnetic field and its interrupting ability. Proc. IEE, 126(4):313, 1979. doi:10.1049/piee.1979.0079.

[12] T. Bonicelli, A. DeLorenzi, D. Hrabal, R. Piovan, E. Saches, E. Sapietro, and S. R. Shaw. The European development of a full scale switching unit for the ITER switching and discharging networks. Fusion Eng. Design, 75:193, 2005. doi:10.1016/j.fusengdes . 2005.06. 225.

[13] A. Zamengo. Operational experience of the $50 \mathrm{kA}-35 \mathrm{kV}$ RFX-mod DC-current interruption system. Proc. 28th ISDEIV, 2:543-546, 2018.

[14] P. G. Slade. The Vacuum Interrupter: Theory Design and Application. CRC Press, Boca Raton, 2008.
[15] R. Renz. Vacuum Interrupters. In Vacuum Electronics: Components and Devices, chapter 8. Springer, 2008.

[16] N. Wenzel, A. Lawall, U. Schümann, and S. Wethekam. Combined experimental and theoretical study of constriction threshold of large-gap AMF vacuum arcs. Proc. 26th ISDEIV, 1:193, 2014.

[17] J. Kusserow and R. Renz. Method for opening the contact gap of a vacuum interrupter. U.S. Patent 7,334,319, 2008.

[18] J. Yan, Z. Liu, Y. Geng, S. Zhang, and Y. Zhang. Investigation on $\mathrm{X}$-radiation for $126 \mathrm{kV}$ vacuum interrupters. Plasma Sci. Technol., 18(5):577, 2016. doi : 10.1088/1009-0630/18/5/22.

[19] J. Brucher, S. Giere, C. Watier, A. Hessenmüller, and P. E. Nielsen. 3AV1FG - $72.5 \mathrm{kV}$ prototype vacuum circuit breaker (case study with pilot customers). 44th Int. Conf. Large High Voltage Electric Systems, A3:101, 2012.

[20] S. Giere, T. Heinz, A. Lawall, C. Stiehler, E. D. Taylor, N. Wenzel, and S. Wethekam. X-radiation emission of high-voltage vacuum interrupters: Dose control under testing and operating conditions. Proc. 28th ISDEIV, pages 523-526, 2018.

[21] W. Hartmann, A. Hauser, A. Lawall, R. Renz, and N. Wenzel. The 3D numerical simulation of a transient vacuum arc under realistic spatial AMF profiles. Proc. 24th ISDEIV, Braunschweig, 2:285-288, 2010. doi:10.1109/DEIV . 2010.5625791.

[22] N. Wenzel, S. Kosse, A. Lawall, R. Renz, and W. Hartmann. Numerical simulation of multi-component arcs in high-current vacuum interrupters. Proc. 25th ISDEIV, 2:321-324, 2012. doi:10.1109/DEIV . 2012.6412518. 